\title{
Preferencia alimentaria de arañas Misumenops pallidus (Araneae: Thomisidae) sobre potenciales insectos presa de cultivos de alfalfa
}

\author{
Germán Cheli ${ }^{1}$, Andrea Armendano \& Alda González ${ }^{2}$ \\ CEPAVE, CONICET, Facultad de Ciencias Naturales y Museo, UNLP, Argentina. Calle 2 № 584 (1900) La Plata, \\ Argentina. Fax 054- 221-4232327. \\ 1 chelicera@arnet.com.ar \\ 2 asgonzalez@cepave.com.ar
}

Recibido 30-IX-2002. Corregido 15-VII-2004. Aceptado 08-XII-2004.

\begin{abstract}
Feeding preferences of the spider Misumenops pallidus (Araneae: Thomisidae) on potential prey insects from alfalfa crops. The spider Misumenops pallidus (Thomisidae) is commonly found in alfalfa crops. We studied its predatory preferences on potential insect prey, particularly regarding agroecosystems pests. Two kinds of tests were done under normal laboratory conditions: simultaneous presentation of prey $(n=215)$ and alternative prey test $(n=45)$. The spiders preferred insects that were mobile, small, without defensive glands and with thin exoskeletons. According to the amount of prey consumed, we established four predation levels: high ( $>55 \%$, on adult Drosophila melanogaster flies); intermediate (30\%-55\% on the defoliator larvae of Rachiplusia $n u$ and adult heteropterans: Horciasinus argentinus and Halticus spegazzinii); and low (10\%-30\% on the chrysomelids Colapsis sp. and Diabrotica speciosa). The pentatomid Piezodorus guildinii, the curculionid Naupactus sp. and the aphid Acyrthosiphom pisum were not accepted as food. Once the spider captured a prey item it did not accept another, independently of prey item species ( $82 \%$ of trials). Rev. Biol. Trop. 54(2): 505513. Epub 2006 Jun 01.
\end{abstract}

Key words: araneae, Thomisidae, Misumenops pallidus, predation, prey preference, alfalfa.

El Manejo Integrado de Plagas (MIP) sostiene que el control biológico surge como resultado de complejas interacciones a nivel de la comunidad. En este marco, la utilización de enemigos naturales es uno de los pilares del MIP, donde los depredadores juegan un papel preponderante. En la última década ha surgido un creciente interés por los depredadores generalistas, especialmente por las arañas que constituyen un grupo resistente que las convierte en los depredadores más abundantes en los ecosistemas terrestres (Rinaldi 1998). Las arañas son en agroecosistemas, el componente dominante de los enemigos naturales limitantes de las poblaciones de herbívoros (Greenstone 1999, Greenstone y Sunderland 1999, Sunderland y Greenstone 1999). Las arañas son numéricamente importantes y conforman un grupo diverso y exitoso, lo que se demuestra en la diversidad de comportamientos que presentan y hábitats ocupados, en la potencialidad de capturar un número mayor de presas que las consumidas y en la riqueza específica (Nyffeler et al. 1987, 1992, Provencher y Riechert 1994, Riechert y Lawrence 1997, Riechert y Maupin 1998, Riechert 1999). La dieta de las arañas, a pesar de ser generalistas, puede llegar a ser muy restricta cuando un tipo de presa es ofrecido en grandes densidades, como suele ocurrir con ciertas plagas en los monocultivos.

En los últimos 35 años, experimentos en cultivos, han probado la capacidad de las 
arañas en reducir las poblaciones de algunos insectos plaga y consecuentemente el daño que causan (Ito et al. 1962, Luczak 1979, Mansour et al. 1980, Mansour y Whitcomb 1986, Mansour 1987, Oraze y Grigarik 1989, Wise 1993, Riechert y Lawrence 1997, Beckerman et al. 1997, Greenstone 1999, Marshall y Rypstra 1999, Rypstra et al. 1999, Marshall et al. 2002).

Teniendo en cuenta las complejas interacciones que existen a nivel de la comunidad, resulta de importancia el rol que cumplen las arañas al complementar su accionar con el de otros enemigos naturales (Sunderland 1999), que junto con la diversificación del hábitat, debe ser considerado al momento de aumentar la sustentabilidad en producciones agrícolas.

La alfalfa (Medicago sativa L.) es una de las especies forrajeras de mayor importancia en la Argentina. Actualmente se cultiva en todo el país, principalmente en la región pampeana y constituye una excelente fuente de proteínas para el ganado. Es un cultivo perenne (con cuatro a seis años de vida útil), con una gran cobertura vegetal, que provee un ambiente estable y favorable para numerosos organismos. Más del $80-90 \%$ del daño a los alfalfares argentinos es causado por tres grupos de plagas primarias, las orugas defoliadoras y cortadoras, los gorgojos y los pulgones (Harcourt et al. 1986). En el presente trabajo se analizó el accionar depredador de Misumenops pallidus (Keyserling) frente a diferentes potenciales insectos presas en cultivos de alfalfa.

\section{MATERIAL Y MÉTODOS}

De muestreos periódicos realizados con antelación al presente trabajo, en diferentes cultivos de alfalfa del partido de La Plata (Buenos Aires, Argentina), resultó que la familia Thomisidae fue la más abundante y $M$. pallidus la especie más común, por lo que se la eligió para realizar el estudio.

Se trabajo con hembras adultas con siete días de ayuno, de 8-10 mm de tamaño, recolectadas en tres cultivos de alfalfa ubicados en la localidad de los Hornos (La Plata, Argentina).
La recolección se efectuó con una red de arrastre de 40 centímetros de diámetro y conjuntamente, con la misma metodología, se muestreó la fauna acompañante, para ser utilizada como potenciales presas en los ensayos, cubriendo los órdenes de insectos más representativos en el campo (Cuadro 1).

Se realizaron dos tipos de prueba: Prueba de presentación simultánea de presas y Prueba de presa alternativa (Jackson y Li 1998).

Los ensayos se llevaron a cabo en el laboratorio con un fotoperíodo de $12 \mathrm{~L} / 12 \mathrm{~N}$ y una temperatura media de $25^{\circ} \mathrm{C}$. Se utilizaron cajas de Petri plásticas de $9 \mathrm{~cm}$ de diámetro por $2 \mathrm{~cm}$ de alto, con un orificio en su tapa superior para ingresar, con el menor stress, a las presas y depredadores (Fig. 1). Se realizaron un total de 260 ensayos donde las presas fueron tomadas de a pares (Cuadro 2).

Prueba de presentación simultánea de presas $(n=215)$ : se introdujo un ejemplar de una presa en la caja, a los diez minutos se agregó un ejemplar de otra especie de presa y transcurridos otros diez minutos un ejemplar de $M$. pallidus. Se consideró el comienzo del ensayo a partir de la introducción de la araña, y su finalización cuando esta última depredó una presa, o transcurridos 30 min desde su ingreso.

Prueba de presa alternativa $(n=45)$ : se introdujo un ejemplar de $M$. pallidus en la caja y se le agregó un ejemplar de una presa (A). Se esperó a que la araña se esté alimentando y se introdujo un ejemplar de una segunda presa (B)

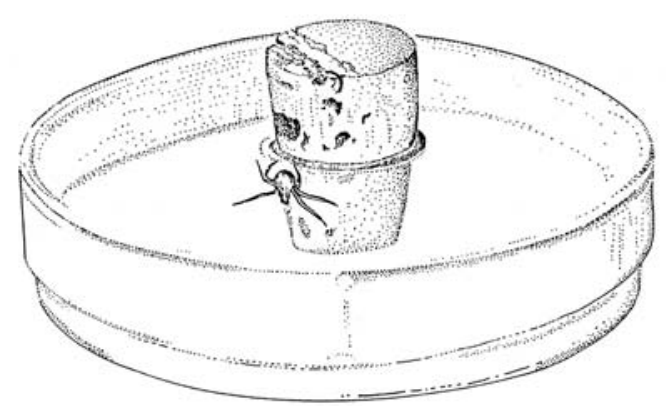

Fig. 1. Caja de Petri acondicionada para los ensayos.

Fig. 1. Petri dish set for the tests. 
CUADRO 1

Presas utilizadas en los ensayos de predación de Misumenops pallidus en el laboratorio

TABLE 1

Prey species used in the predation tests of Misumenops pallidus in the laboratory

\begin{tabular}{|c|c|c|c|c|}
\hline Orden & Familia & Género / especie & Estado de desarrollo & $\begin{array}{l}\text { Tamaño relativo } \\
\text { a } M \text {. Pallidus }\end{array}$ \\
\hline \multirow[t]{2}{*}{ Coleoptera } & Curculionidae & Naupactus sp. & Adulto & Igual a mayor \\
\hline & Chrysomelidae & $\begin{array}{l}\text { Diabrotica speciosa } \\
\text { Colaspis sp. }\end{array}$ & $\begin{array}{l}\text { Adulto } \\
\text { Adulto }\end{array}$ & $\begin{array}{l}\text { Menor a igual } \\
\text { Menor a igual }\end{array}$ \\
\hline Lepidoptera & Noctuidae & Rachiplusia $n u$ & Larva & Mayor \\
\hline Homoptera & Aphididae & Acyrthosiphom pisum & Adulto & Menor \\
\hline \multirow[t]{2}{*}{ Heteroptera } & Pentatomidae & Piezodorus guildinii & Adulto y ninfa IV & Igual a mayor \\
\hline & Miridae & $\begin{array}{l}\text { Horciasinus argentinus } \\
\text { Halticus spegazzinii }\end{array}$ & $\begin{array}{l}\text { Adulto } \\
\text { Adulto }\end{array}$ & $\begin{array}{l}\text { Menor } \\
\text { Menor }\end{array}$ \\
\hline Diptera & Drosophilidae & Drosophila melanogaster & Adulto & Menor \\
\hline
\end{tabular}

diferente de la primera, considerándose éste el comienzo del experimento. En otro ensayo, a otra araña alimentándose de la especie "B" se la contactó con un ejemplar de especie "A". El ensayo finalizó cuando la primera presa fue descartada por preferir la segunda o transcurridos 30 min desde el comienzo.

Los resultados fueron analizados mediante un prueba de bondad de ajuste $\chi^{2}(\mathrm{p}<0.05)$ (Sokal y Rohlf 1986).

\section{RESULTADOS}

De la realización del prueba de presentación simultánea de presas (Cuadro 2, Fig. 2) surge que los dípteros (Drosophila melanogaster) conforman claramente la presa más depredada por M. pallidus, seguidos en segundo lugar por un grupo conformado por heterópteros Miridae (Horciasinus argentinus y Halticus spegazzinii) y lepidópteros Noctuidae (Rachiplusia $\mathrm{nu}$ ). El tercer lugar lo ocuparía otro grupo conformado por coleópteros crisomélidos (Diabrotica speciosa y Colaspis sp.) y finalmente se ubicaría el grupo de los áfidos (Acyrthosiphom pisum), curculiónidos
(Naupactus sp.) y heterópteros Piezodorus guildinii. De éstos, los dos últimos conformarían dentro de los ítems alimentarios ofrecidos, los que nunca fueron consumidos, mientras que los áfidos alguna vez fueron elegidos. No obstante se los ubica también en este nivel porque solo fueron depredados, en dos oportunidades de los 34 ensayos realizados correspondientes a la primera prueba y al ser ofrecida otra presa alternativa

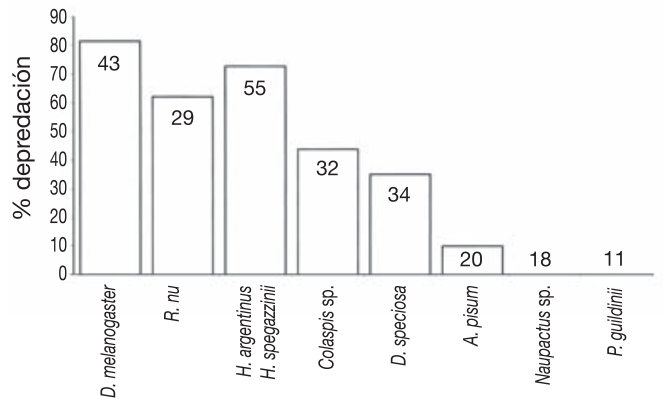

Fig. 2. Depredación de Misumenops pallidus frente a diferentes presas ofrecidas. Los números en cada barra indican el tamaño muestral.

Fig. 2. Misumenops pallidus predation on several prey items. Bar numbers: sample size. 
CUADRO 2

Prueba de presentación simultánea de presas a Misumenops pallidus en el laboratorio

TABLE 2

Test of simultaneous presentation of prey to Misumenops pallidus in the laboratory

Pares de presas

Presa 1
D. melanogaster
D. melanogaster
D. melanogaster
D. melanogaster
D. melanogaster
D. melanogaster

Miridae

Miridae

Miridae

Miridae

Miridae

Miridae

Rachiplusia nu

Rachiplusia nu

Rachiplusia $n u$

Colaspis sp.

Colaspis sp.

Colaspis sp.

Colaspis sp.

Diabrotica speciosa

Diabrotica speciosa

Diabrotica speciosa

Naupactus sp.
Presa 2

Colaspis sp.

Naupactus sp.

Rachiplusia nu

Miridae

Acyrthosiphom pisum

Diabrotica speciosa

Colaspis sp.

Naupactus sp.

Rachiplusia nu

Acyrthosiphom pisum

Diabrotica speciosa

Piezodorus guildinii

Colaspis sp.

Naupactus sp.

Diabrotica speciosa

Naupactus sp.

Acyrthosiphom pisum

Diabrotica speciosa

Piezodorus guildinii

Naupactus sp.

Acyrthosiphom pisum

Piezodorus guildinii

Acyrthosiphom pisum
Prefiere
presa 1

10 *

$4 * *$

4

$7 * *$

$6 *$

$4 *$

2

$8 *$

6

$10 *$

$8 * *$

3

3

$4 * *$

5

0

0

4

4

2

2

$4 *$

0
Prefiere
presa 2

No come

$\begin{array}{ll}0 & 11 \\ 1 & 5 \\ 0 & 7 \text { n.s. } \\ 1 * * & 11 \\ 0 & 7 \\ 0 & 4 \\ 5 & 11 \text { n.s. } \\ 4 & 12 \\ 2 & 11 \text { n.s. } \\ 1 & 11 \\ 4 & 13 \\ 6 * & 9\end{array}$

7 n.s.

5

9

9

5

18

7 n.s.

7

5 n.s.

4

6

$* \mathrm{p}<.05 ; * * \mathrm{p}<.1 ;$ n.s. no significativo.

(segunda prueba), en forma casi inmediata, la araña los descartó.

Teniendo en cuenta la depredación de $M$. pallidus por las diferentes presas ofrecidas se establecieron los siguientes niveles: Depredación
Máxima, Depredación Intermedia, Depredación Mínima y No Depredados (Cuadro 3).

Por otro lado se observó, como resultado de la aplicación de esta misma prueba, que a medida que decrece la preferencia por la presa, 
CUADRO 3

Niveles de depredación de Misumenops pallidus frente a diferentes presas ofrecidas en el laboratorio

TABLE 3

Predation levels of Misumenops pallidus on prey items offered in the laboratory

Nivel de depredación

Depredación máxima $(>55 \%)$

Depredación intermedia (30-55\%)

Depredación mínima (10-30\%)

No depredados $(<10 \%)$
Presas

Diptera, Drosophilidae: Drosophila melanogaster

Heteroptera, Miridae:

Horciasinus argentinus

Halticus spegazzinii

Lepidoptera, Noctuidae: Rachiplusia $n u$

Coleoptera, Chrysomelidae: Colaspis sp.

Diabrotica speciosa

Homoptera, Aphididae: Acyrthosiphom pisum

Coleoptera, Curculionidae: Naupactus sp.

Heteroptera, Pentatomidae: Piezodorus guildinii disminuye el consumo hasta convertirse en cero (nivel No depredado), es decir se incrementa la decisión de M. pallidus de no consumir ninguna presa (Fig. 3). Esto confirmaría los niveles de depredación señalados anteriormente.

Los resultados de los 45 ensayos efectuados de la prueba de presas alternativas mostraron que raramente $M$. pallidus deja la presa que esta consumiendo para tomar otra. Solamente en ocho experiencias descartó la presa que estaba consumiendo y optó por la ofrecida en segundo término (18\%) (Cuadro 4). De estos ocho ensayos, en tres las presas descartadas correspondieron a áfidos (Nivel No depredado < 10\%).

\section{DISCUSIÓN}

El nivel de depredación de $M$. pallidus se relacionó con el tamaño de las presas ofrecidas, el grosor de sus cutículas, la presencia de defensas químicas y la movilidad, mostrando una mayor predilección por las presas de dimensiones menores, de exoesqueletos más delgados, sin sustancias repelentes para los

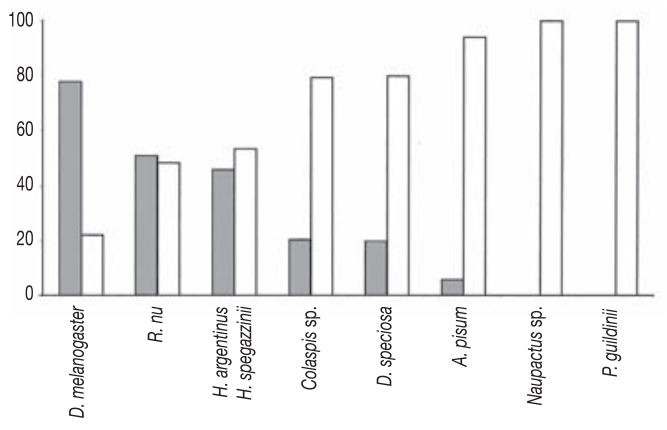

$\square$ con consumo de presa* $\square$ sin consumo de presa**

Fig. 3. Porcentaje de consumo por Misumenops pallidus en función de la calidad de las presas (* consumo de una presa determinada, ** no consumo y/o consumo de una presa alternativa).

Fig. 3. Percentage of prey consumption by Misumenops pallidus according to the prey quality (* particular prey consumed, ** prey not consumed, or alternative prey consumed).

depredadores y las de mayor movilidad. De nuestras experiencias surge que:

1- M. pallidus muestra una actitud de depredación positiva hacia moscas adultas, 
CUADRO 4

Prueba de presas alternativas ofrecidas a Misumenops pallidus en el laboratorio

TABLE 4

Test of alternative prey items offered to Misumenops pallidus in the laboratory

Presa en consumo

Rachiplusia nu

Rachiplusia $n u$

Drosophila melanogaster

Drosophila melanogaster

Rachiplusia nu

Colaspis sp.

Colaspis sp.

Miridae

Rachiplusia $n u$

Acyrthosiphom pisum

Miridae

Miridae

Miridae

Colaspis sp.

Diabrotica speciosa

Piezodorus guildinii

Diabrotica speciosa

Acyrthosiphom pisum

Rachiplusia nu

Drosophila melanogaster
Presa ofrecida

Naupactus sp.

Colaspis sp.

Rachiplusia $n u$

Colaspis sp.

Drosophila melanogaster

Rachiplusia un

Drosophila melanogaster

Acyrthosiphom pisum

Acyrthosiphom pisum

Miridae

Drosophila melanogaster

Rachiplusia $n u$

Colaspis sp.

Miridae

Drosophila melanogaster

Drosophila melanogaster

Colaspis sp.

Drosophila melanogaster

Miridae

Miridae
Toma presa ofrecida

No toma presa ofrecida

\begin{tabular}{|c|c|}
\hline & 2 \\
\hline & 1 \\
\hline & 2 \\
\hline & 5 \\
\hline & 2 \\
\hline & 2 \\
\hline & 3 \\
\hline & 1 \\
\hline & 2 \\
\hline 2 & \\
\hline 4 & 5 \\
\hline 1 & 2 \\
\hline & 2 \\
\hline & 1 \\
\hline & 3 \\
\hline & 1 \\
\hline & 1 \\
\hline 1 & \\
\hline & 1 \\
\hline & 1 \\
\hline 8 & 37 \\
\hline
\end{tabular}

$R . n u$, si bien presenta una cutícula delgada y sin glándulas repelentes, su tamaño y escasa movilidad dificulta la manipulación por parte de la araña, mientras que los heterópteros adultos a pesar de ser de pequeño tamaño y sin defensas químicas, presentan una cutícula de mayor grosor y menor movilidad que los dípteros.

3- Frente a los coleópteros crisomélidos (Colaspis sp. y D. speciosa), M. pallidus mostró un nivel de Depredación Mínima 
(10\%-30\%). Las presas presentan lento desplazamiento, un tamaño semejante o mayor y un exoesqueleto de gran grosor lo que complicaría la depredación y su consumo por parte de la araña.

4- Finalmente, M. pallidus manifestó una actitud de depredación negativa por los coleópteros curculiónidos (Naupactus sp.), por los hemípteros pentatómidos ( $P$. guildinii) y por los áfidos (A. pisum). En el primer caso se trata de presas pequeñas pero con exoesqueletos gruesos lo que dificulta la inoculación del veneno y con poca movilidad lo que no favorece el encuentro con la araña. En los otros dos casos la depredación negativa está relacionada con la presencia de glándulas pestilentes, que resultan una buena defensa frente a los depredadores (Richards y Davies 1984, Nentwig 1986). Estos tres grupos corresponden al nivel de No Depredados $(<10 \%)$.

5- En el $82 \%$ de los casos, independientemente del tipo de presa considerada, M. pallidus una vez que decide cazar y lo consigue, no cambia a su presa por otra.

Por ser las arañas depredadores generalistas que atacan a sus presas con relación a la frecuencia de encuentros, cuanto mayor sea la densidad de una especie presa (como ocurre en los monocultivos), mayor será la proporción de ataques hacia dicha presa y por ende más restricta resultará su dieta (Riechert y Lawrence 1997). Coincidiendo con esto Nyffeler y Benz (1988) encontraron que Achaearanea riparia se comporta como depredador monófago de hormigas en hábitats donde éstas constituyen las presas más abundantes, y como generalistas en hábitats donde otros grupos de potenciales presas aparecen en gran número. González et al., en un trabajo enviado al Journal of Arachnology, referido a la preferencia y tasa de depredación de M. pallidus sobre insectos plaga de cultivos de soja y realizando experiencias a campo, sostienen que esta araña mostró una clara preferencia positiva hacia las larvas de los defoliadores. En el presente trabajo M. pallidus mostró una depredación intermedia hacia $R$. nu en presencia del resto de presas ofrecidas, pero siendo esta presa uno de los tres grupos de plagas primarias de la alfalfa que ocasionan más del 90\% del daño a los alfalfares argentinos (Lanteri 1994), en el momento de mayor densidad de esta plaga existen más posibilidades del accionar de la araña sobre la misma. Por otro lado, si tenemos en cuenta que los depredadores pueden afectar el impacto de los insectos plaga de forma directa debido al consumo de los mismo, y además de forma indirecta, debido a que la sola presencia de ellos representa un riesgo de depredación de herbívoros, que puede afectar el comportamiento de forrajeo de estas presas (Beckerman et al. 1997), queda de manifiesto la importancia del estudio de las arañas en su rol de enemigos naturales de insectos plaga.

Por otro lado, M. pallidus presenta características favorables para ser tenidas en cuenta como un factor de mortalidad de insectos en el cultivo de alfalfa por ser una especie que coloniza el cultivo desde los comienzos de su desarrollo y se dispersa ampliamente (A. Armendano com. pers.), lo que permite la depredación sobre insectos fitófagos aún cuando sus densidades en el cultivo sean bajas, retardando el incremento poblacional y porque tiene superposición de generaciones donde los subadultos y adultos (que tienen el mayor consumo) son proporcionalmente importantes en todo momento.

Esto sugiere que tácticas de conservación de estos depredadores generalistas compatibles con otras estrategias de control, son aconsejables en el manejo integrado de plagas en agroecosistemas.

\section{RESUMEN}

Se analizó la preferencia alimentaria de la araña Misumenops pallidus frente a diferentes potenciales insectos presas, algunos de los cuales son plaga en cultivos agrícolas. Se efectuaron pruebas de presentación simultánea de presas $(n=215)$ y de presa alternativa $(n=45)$, en condiciones normales de laboratorio. M. pallidus mostró predilección por insectos móviles, pequeños, de exoesqueleto delgado y sin glándulas repelentes. Se establecieron 
cuatro niveles de depredación. El grado máximo (> 55\%) correspondió a las moscas adultas Drosophila melanogaster. Hubo depredación intermedia (30\%-55\%) de larvas desfoliadoras (Rachiplusia nu) y heterópteros adultos (Horciasinus argentinus y Halticus spegazzinii); y depredación mínima (10\%-30\%) de los crisomélidos Colaspis sp. y Diabrotica speciosa. Los pentatómidos Piezodorus guildinii, los curculiónidos Naupactus sp. y los áfidos Acyrthosiphom pisum, no fueron depredados. En el $82 \%$ de los casos, una vez que $M$. pallidus logra cazar su presa, no la cambia por otra (independientemente del tipo de presa considerada).

Palabras clave: araneae, Thomisidae, Misumenops pallidus, depredación, preferencia de presas, alfalfa.

\section{REFERENCIAS}

Beckerman, A.P., M. Uriarte \& O. J. Schmitz. 1997. Experimental evidence for a behavior-mediated trophic cascade in a terrestrial food chain. Proc. Natl. Acad. Sci. USA 94: 10735-10738.

Greenstone, M.H. 1999. Spider predation: how and why we study it. J. Arachnol. 27: 333-342.

Greenstone, M.H. \& K.D. Sunderland. 1999. Why a symposium on spiders in agroecosystems now? J. Arachnol. 27: 267-269.

Harcourt, D.C., J.R. Aragón \& R. González. 1986. Plagas de la alfalfa. In C. Bariggi, B.L. Marble, C.D. Itria \& J.M. Brun (eds.). Investigación, tecnología y producción de alfalfa. Colección Científica del INTA, Tomo XXII, Buenos Aires, Argentina. 488 p.

Ito, Y., K. Miyashita \& K. Sekiguchi. 1962. Studies on the predators of the rice crop insect pests using the insecticidal check method. Jpn. J. Ecol. 12: 1-11.

Jackson, R.R. \& D. Li. 1998. Prey preferences and visual discrimination ability of Cyrba algerina, an araneophagic jumping spiders (Araneae: Salticidae) with primitive retinae. Isr. J. Zool. 44: 227-242.

Lanteri, A. 1994. Bases para el control integrado de los gorgojos de la alfalfa. De La Campana, La Plata, Argentina. 119 p.

Luczak, J. 1979. Spiders in agrocoenoses. Polish Ecol. Stud. 5: 151-200.

Mansour, F. 1987. Effects of pesticides on spiders ocurring on apple and citrus in Israel. Phytoparasitica 15: 43-51.

Mansour, F., D. Rosen, A. Shulov \& H.N. Plaut. 1980. Evaluation of spiders as biological control agents of
Spodoptera littoralis larvae on apple in Israel. Acta Oecol. 1: 225-232.

Mansour, F. \& W.H. Whitcomb. 1986. The spiders of a citrus grove in Israel and their role as biocontrol agents of Ceroplastes floridensis (Homoptera: Coccidae). Entomophaga 31: 269-276.

Marshall, S.D. \& A. Rypstra. 1999. Habitat selection determines the colonization of two soybean agroecosytems by two wolf spiders (Araneae, Lycosidae). Environ. Entomol. 28: 1052-1059.

Marshall, S.D., D.M. Pavuk \& A.L. Rypstra. 2002. A comparative study of phenology and daily activity patterns in the wolf spiders Pardosa milvina and Honga helluo in soybean agroecosystems in Southwestern Ohio (Araneae, Lycosidae). J. Arachnol. 30: 503-510.

Nentwig, W. 1986. The Prey of Spiders, p. 249-263. In W. Nentwig (eds.). Ecophysiology of Spiders. Springer, Berlin-Heidelberg, Alemania.

Nyffeler, M., D.A. Dean \& L. Sterling. 1987. Evaluation of the importance of the striped lynx spider, Oxyopes salticus (Araneae: Oxyopidae), as a predator in Texas cotton. Environ. Entomol. 16: 1114-1123.

Nyffeler, M.D.A. \& G. Benz. 1988. Prey analysis of the spider Archaearanea riparia (Blackw) (Araneae, Theridiidae) a generalist predator in winter wheat fields. J. Appl. Entomol. 106: 425- 431.

Nyffeler, M., D.A. Dean \& L. Sterling. 1992. Diets, feeding specialization, and predatory role of two lynx spiders, Oxyopes salticus and Peucetia viridans (Araneae: Oxyopidae) in Texas cotton agroecosystem. Environ. Entomol. 21: 1457-1465.

Oraze, M.J. \& A.A. Grigarick. 1989. Biological control of aster leafhopper (Homoptera: Cicadellidae) and midges (Diptera: Chironomidae) by Pardosa ramulosa (Araneae: Lycosidae) in California rice fields. J. Economic Entomol. 82: 745-749.

Provencher, L. \& S.E. Riechert. 1994. Model and field test of prey control effects by spider assemblages. Environ. Entomol. 23: 1-17.

Richards \& Davies. 1984. Tratado de Entomología. Imm. Vol. 2 Clasificación y Biología. Omega, Buenos Aires, Argentina. 319 p.

Riechert, S.E. \& K. Lawrence. 1997. Test for predation effects of single versus multiple species of generalist predators: Spiders and their insect prey. Entomol. Exp. Appl. 4: 147-155.

Riechert, S.E. \& J. Maupin. 1998. Spiders effects on prey: tests for superfluous killing in five web-builders, p. 203-210. Proc. 17 $7^{\text {th }}$ European Coll. Arachnol. Edinburgo, Escocia. 
Riechert, S. E. 1999. The hows and whys of successful pest suppression by spiders: insights from case studies. J. Arachnol. 27: 387- 396.

Rinaldi, I. 1998. Aranhas en agroecosistemas do Brasil. Anais do VI SINCOBIOL. Río de Janeiro, Brasil. p. $384-388$

Rypstra, A., P. Carter, R. Balfour \& S. Marshall. 1999 Architectural features of agricultural habitats and their impact on the spider inhabitants. J. Arachnol. 27: 371-377.
Sokal, R. \& J. Rohlf. 1986. Introducción a la Bioestadística. Reverté, Buenos Aires, Argentina. 363 p.

Sunderland, K. 1999. Mechanisms underlying the effects of spiders on pest populations. J. Arachnol. 27: 308-316.

Sunderland, K.D. \& M.H. Greenstone. 1999. Summary and future directions for research on spiders in agroecosystems. J. Arachnol. 27: 397-400.

Wise, D.H. 1993. Spiders in Ecological webs. Cambridge Studies in Ecology, Cambridge University, Cambridge, Reino Unido. 328 p. 
